\title{
Molluscum Contagiosum Superimposed on Lymphangioma Circumscriptum
}

\author{
Anna Kim, MD; Jiehyun Jeon, MD, PhD; Hae Jun Song, MD, PhD; Yoo Sang Baek, MD, PhD
}

\section{PRACTICE POINTS}

- Lymphangioma circumscriptum (LC) is a benign malformation of the lymphatic system that can be misdiagnosed as molluscum contagiosum (MC).

- Secondary infection of LC is common, with Staphylococcus aureus being the most common entity, but MC virus also can be secondarily infected.

To the Editor:

Lymphangioma circumscriptum (LC) is a benign malformation of the lymphatic system. ${ }^{1}$ It is postulated to arise from abnormal lymphatic cisterns, and it grows separately from the normal lymphatic system. These cisterns are connected to malformed dermal lymphatic channels, and the contraction of smooth muscles lining cisterns will cause dilatation of connected lymphatic channels in the papillary dermis due to back pressure, ${ }^{1,2}$ which causes a classic LC manifestation characterized by multiple translucent, sometimes red-brown, small vesicles grouped together. Lymphangioma circumscriptum can be difficult to differentiate from molluscum contagiosum (MC) due to the similar morphology. ${ }^{1}$ We present a notable case of $\mathrm{MC}$ superimposed on LC.

A 6-year-old girl presented with multiple grouped, clear, vesicular papules on the right buttock of 18 months' duration. Some of the papules showed tiny whitish pearllike particles on the top (Figure 1). Similar lesions were not present elsewhere on the body. She had no underlying disease and did not have a history of procedure,
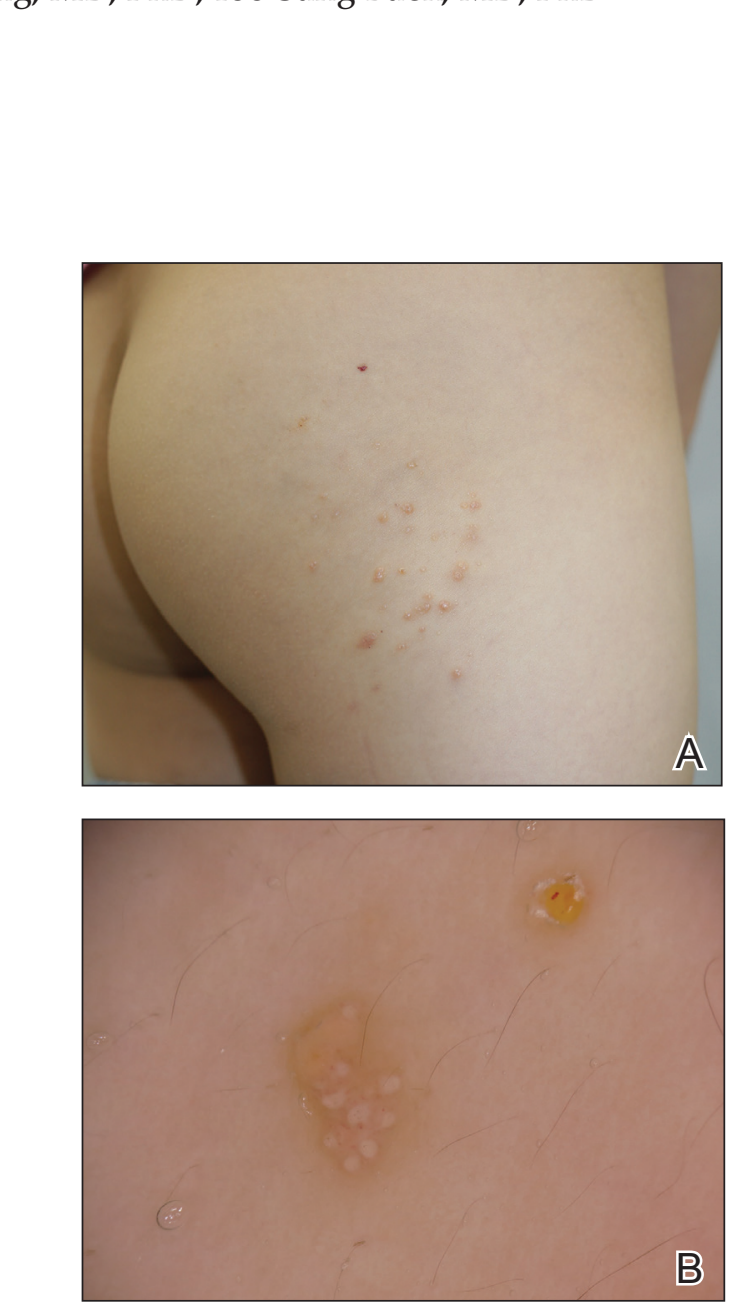

FIGURE 1. A, Multiple grouped, clear, vesicular papules on the right buttock. Tiny whitish pearl-like particles were observed on top of the selected vesicular papules. B, Dermoscopy revealed whitish pearl-like structures and yellowish lacunae with minor vascular structures (original magnification $\times 10$ ).

From the Department of Dermatology, Guro Hospital, Korea University College of Medicine, Seoul.

The authors report no conflict of interest.

Correspondence: Yoo Sang Baek, MD, PhD, Department of Dermatology, Guro Hospital, Korea University College of Medicine, 148 Gurodong-ro, Guro-gu, Seoul 08308, Korea (baekyoosang@gmail.com).

doi: $10.12788 /$ cutis.0376 
edema, or malformation of the lower extremities. Histopathology from one of the lesions showed dilated cystic lymphatic spaces in the papillary dermis lined with flattened endothelium and cup-shaped downward proliferation of the epidermis with presence of large intracytoplasmic inclusion bodies-features of both LC and MC (Figure 2). We waited 4 additional months for the MC lesions to self-resolve, but they persisted. The patient's mother strongly requested for their removal, and the residual MC lesions were carefully removed by $\mathrm{CO}_{2}$ laser. To prevent unnecessary physical damage to underlying LC lesions and minimize scarring, we opted to use the $\mathrm{CO}_{2}$ laser and not simple curettage. She currently is under periodic observation with no signs of clinical recurrence of $\mathrm{MC}$, but the LC lesions naturally persisted.

Due to its vesicular and sometimes warty appearance, LC can sometimes be hard to differentiate from MC. In one report, a vesicular plaquelike lesion on the trunk initially was misdiagnosed and treated as MC but was histologically confirmed as LC several years later. ${ }^{3}$ Our case demonstrates the coexistence of MC and LC. Although this phenomenon may be coincidental, we have not noticed any additional MC lesions on the body and MC only existed over the LC lesions, implying a possible pathophysiologic relationship. It is unlikely that $\mathrm{MC}$ might have preceded the development of LC. Although acquired LC exists, it has mostly been reported in the genital region of patients with conditions leading to lymphatic obstruction such as surgery, radiation therapy, malignancy, or serious infections. ${ }^{4}$ Because our patient developed lesions at an early age without any remarkable medical history, it is likely that she had congenital LC that was secondarily infected by the MC virus. Vesicular lesions in LC are known to rupture easily and may serve as a vulnerable entry site for pathogens. Subsequent secondary bacterial infections are common, with Staphylococcus aureus being the most prominent entity. ${ }^{1}$ However, secondary viral infection rarely is reported. It is possible that the abnormally dilated lymphatic channels of LC that lack communication with the normal lymphatic system

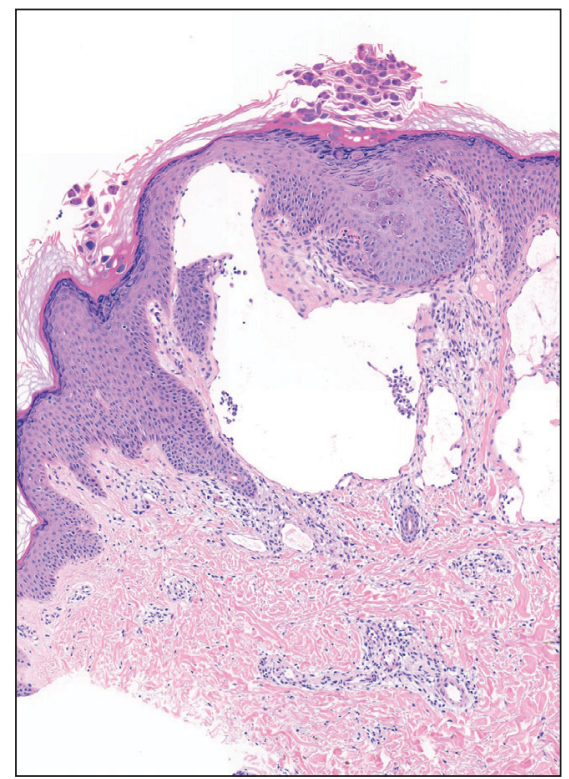

FIGURE 2. Multiple dilated cystic lymphatic spaces in the papillary dermis lined with flattened endothelium. Cup-shaped downward proliferations of the epidermis with presence of large intracytoplasmic inclusion bodies also were observed (H\&E, original magnification $\times 100$ ).

have contributed to an LC site-specific vulnerability to $\mathrm{MC}$ virus. Further studies and subsequent reports are required to confirm this hypothesis.

\section{REFERENCES}

1. Patel GA, Schwartz RA. Cutaneous lymphangioma circumscriptum: frog spawn on the skin. Int J Dermatol. 2009;48:1290-1295. doi:10.1111 /j.1365-4632.2009.04226.x

2. Fatima $\mathrm{S}$, Uddin $\mathrm{N}$, Idrees $\mathrm{R}$, et al. Lymphangioma circumscriptum: clinicopathological spectrum of 29 cases. J Coll Physicians Surg Pak. 2015;25:658-661. doi:09.2015/JCPSP.658661

3. Patel GA, Siperstein RD, Ragi G, Schwartz RA. Zosteriform lymphangioma circumscriptum. Acta Dermatovenerol Alp Pannonica Adriat. 2009;18:179-182.

4. Chang MB, Newman CC, Davis MD, et al. Acquired lymphangiectasia (lymphangioma circumscriptum) of the vulva: clinicopathologic study of 11 patients from a single institution and 67 from the literature. Int J Dermatol. 2016;55:E482-E487. doi:10.1111/ijd.13264 